Europhysics Letters

PREPRINT

\title{
Switching the current through molecular wires with Gaussian laser pulses
}

\author{
Ulrich Kleinekathöfer, GuangQi Li, Sven Welack and Michael Schreiber \\ Institut für Physik, Technische Universität Chemnitz, 09107 Chemnitz, Germany
}

PACS. 73.63.Nm - Quantum wires.

PACS. 78.67.Lt - Quantum wires.

PACS. 33.80.-b - Photon interactions with molecules.

\begin{abstract}
The influence of Gaussian laser pulses on the transport through molecular wires is investigated within a tight-binding model for spinless electrons including correlation. Motivated by the phenomenon of coherent destruction of tunneling for monochromatic laser fields, situations are studied in which the maximum amplitude of the electric field fulfills the conditions for the destructive quantum effect. It is shown that, as for monochromatic laser pulses, the average current through the wire can be suppressed. For parameters of the model, which do not show a net current without any optical field, a Gaussian laser pulse can establish a temporary current. In addition, the effect of electron correlation on the current is investigated.
\end{abstract}

Introduction. - In recent years many groups have been working on making the vision of molecular electronics reality [1,2]. This bottom-up approach for electronic devices has certain advantages over standard top-down approaches which are mainly used these days. In molecular electronics the transport is through single molecules or molecular aggregates and has therefore to be treated quantum mechanically. This quantum nature makes it of course more complicated to determine, for example, the current-voltage characteristics than in classical theories. But at the same time a quantum treatment offers certain advantages and especially the possibility of constructive or destructive interference effects.

In the current letter we focus on the electron transport through a molecular wire which is coupled to two leads acting as electron source and drain. Many theories of quantum transport utilize the non-equilibrium Green's function approach [3] which is formally exact within the lead-wire coupling. But because of the dependence of Green's functions on two time arguments it is rather difficult to determine the current without any further approximations, as for example, the wide-band limit. Another possible route is to treat the lead-wire coupling perturbatively and derive quantum master equations for the electron dynamics in the wire and the current through the wire [4-7]. In addition to the wire-lead coupling a laser field can be coupled to the wire and/or the leads. This would possibly allow for an ultrafast opto-electronic device. First experimental [8] and theoretical $[4,7]$ investigations in this direction have been performed. Most of the theoretical studies are based on a master equation approach since in this formalism allows easily to include time-dependent laser fields. For time-periodic fields the Floquet theory can be employed [4]. Recently the present authors derived a formalism which

(C) EDP Sciences 


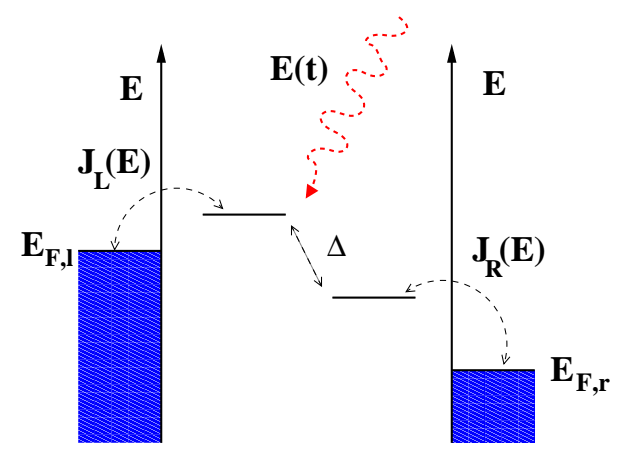

Fig. 1 - Sketch of the two-site system defined in the text.

treats the laser-wire interaction exactly for an arbitrary time-dependence of the field [7]. This approach has been developed in analogy to the treatments in dissipative quantum dynamics, but instead of a bosonic heat bath in dissipation theories the leads are treated as fermionic reservoirs. In addition, the system-bath coupling operator allows for particle exchange in the case of molecular wires while in dissipative theories only energy exchange is allowed. The present approach makes use of the spectral decomposition of the spectral density $J(\omega)$ first introduced by Meier and Tannor [9]. These authors applied a time-nonlocal formalism based on the Nakajima-Zwanzig equation. Later time-local theories based on the same trick have been developed [10,11]. For molecular wires these two versions of quantum master equations have been compared in Ref. [12].

Grossmann et al. $[13,14]$ showed in their pioneering work that periodically driven quantum systems can exhibit the unusual effect of quenching the tunneling dynamics at specific values of field amplitude. This effect was termed coherent destruction of tunneling (CDT). The validity range of this localization of particles by external periodic fields has later been studied in more detail for situations including a bias [15]. For cw-laser fields CDT has already been described for model molecular wires using the Floquet theory [16]. The purpose of this work is to study the CDT for short Gaussian laser pulses which have a high carrier frequency. Earlier studies [7] suggest that the CDT phenomenon survives also for shorter pulse length not just infinite length as in the original CDT studies. In those studies an average current was investigated. For cw-laser fields the CDT appears to be perfect if one measures at intervals separated by the period of the driving field but there are still oscillations within one period. Another way of looking at the effect is by averaging over one period of the periodic driving in the cw case. As will be discussed later, for shorter laser pulses one needs to average over several of the high-frequency oscillations of the carrier frequency.

Model. - As in many quantum dissipation theories, the physical system is separated into the relevant system $H_{S}(t)$, mimicking the wire, and reservoirs $H_{R}$ modeling the leads

$$
H(t)=H_{S}(t)+H_{R}+H_{S R}
$$

with wire-lead coupling $H_{S R}$. The wire consists of $N$ sites coupled to each other by a hopping element $\Delta$ (see Fig. 1). Denoting the creation (annihilation) operator by $c_{n}^{\dagger}\left(c_{n}\right)$ the tightbinding description of the electrons in the molecular wire reads

$$
H_{S}(t)=\sum_{n} E_{n} c_{n}^{\dagger} c_{n}-\Delta \sum_{n}\left(c_{n}^{\dagger} c_{n+1}+c_{n+1}^{\dagger} c_{n}\right)+U \sum_{n} c_{n}^{\dagger} c_{n} c_{n+1}^{\dagger} c_{n+1}-\mu E(t) .
$$


The first term describes the on-site energies, the second term the nearest-neighbor hopping and the third term electron correlation within the wire in a version for spinless particles. The fourth term gives the coupling between the wire and the laser field $E(t)$ whereas for the dipole operator we assume [4]

$$
\mu=e \sum_{n} x_{n}=e \sum_{n} \frac{2 n-N-1}{2} c_{n}^{\dagger} c_{n} .
$$

The environment of the wire consists of two electronic leads that are modeled by two independent reservoirs of uncorrelated electrons in thermal equilibrium. For each lead, the Hamiltonian $H_{R}$ is given by $H_{R}=\sum_{q} \omega_{q} c_{q}^{\dagger} c_{q}$ with $c_{q}^{\dagger}$ and $c_{q}$ creating and annihilating an electron in the corresponding reservoir mode $|q\rangle$ with energy $\omega_{q}$. $\hbar$ is set to unity throughout the paper. Due to the assumed thermal equilibrium of the electronic leads, the occupation expectation values of the reservoir modes are determined by $\left\langle c_{q}^{\dagger} c_{q^{\prime}}\right\rangle=n_{F}\left(\omega_{q}-E_{F}\right) \delta_{q q^{\prime}}$ where $n_{F}$ is the Fermi function and $E_{F}$ the Fermi energy. In further derivations we will only refer to the left lead but the formalism has to be applied to the right lead coupled to the last site $N$ of the wire as well. The coupling of the left electronic lead with the first site of the wire is given by

$$
H_{S R}=\sum_{x=1}^{2} K_{x} \Phi_{x}=\sum_{q}\left(V_{q} c_{1}^{\dagger} c_{q}+V_{q}^{*} c_{q}^{\dagger} c_{1}\right)
$$

with $\Phi_{1}=\sum_{q} V_{q} c_{q}, \Phi_{2}=\sum_{q} V_{q}^{*} c_{q}^{\dagger}, K_{1}=c_{1}^{\dagger}, K_{2}=c_{1}$, and a wire-lead coupling strength $V_{q}$ for each reservoir mode.

As one is normally not interested in the dynamics within the leads but only within the wire, a quantum master equation (QME) based on a second-order perturbation theory in the wire-lead coupling has been developed for the reduced density matrix of the wire $\rho_{S}(t)[7]$

$$
\dot{\rho}_{S}(t)=-i \mathcal{L}_{S}(t) \rho_{S}(t)-\sum_{x x^{\prime}}^{1,2}\left[K_{x}, \Lambda_{x x^{\prime}}(t)-\widehat{\Lambda}_{x x^{\prime}}(t)\right]
$$

with $\mathcal{L}_{S}(t)$ being the Liouville operator applying $H_{S}$ and the auxiliary operators

$$
\begin{aligned}
& \Lambda_{x x^{\prime}}(t)=\int_{t_{0}}^{t} \mathrm{~d} t^{\prime} C_{x x^{\prime}}\left(t-t^{\prime}\right) U_{S}\left(t, t^{\prime}\right) K_{x^{\prime}} \rho_{S}\left(t^{\prime}\right), \\
& \widehat{\Lambda}_{x x^{\prime}}(t)=\int_{t_{0}}^{t} \mathrm{~d} t^{\prime} C_{x^{\prime} x}^{*}\left(t-t^{\prime}\right) U_{S}\left(t, t^{\prime}\right) \rho_{S}\left(t^{\prime}\right) K_{x^{\prime}} .
\end{aligned}
$$

Here we employed the definitions $U_{S}\left(t, t^{\prime}\right)=T_{+} \exp \left(-i \int_{t^{\prime}}^{t} \mathrm{~d} \tau \mathcal{L}_{S}(\tau)\right)$ and the reservoir correlation functions $C_{x x^{\prime}}(t)=\operatorname{tr}_{R}\left\{\mathrm{e}^{i H_{R} t} \Phi_{x} \mathrm{e}^{-i H_{R} t} \Phi_{x^{\prime}} \rho_{R}\right\}$ with the reservoir density matrix $\rho_{R}$. Using this form of the QME has the advantages over the standard Redfield approach [17] that the memory terms are included and that the wire-laser coupling is still treated exactly within the dipole approximation.

For the QME all the external properties of the fermionic reservoir are described by a single quantity, namely the spectral density $J_{R}(\omega)=\sum_{q} \pi\left|V_{q}\right|^{2} \delta\left(\omega-\omega_{q}\right)$. The sum becomes a smooth function for a dense spectrum of the reservoir modes. Now we use the trick of a numerical decomposition of the spectral density $[7,9]$

$$
J_{R}(\omega)=\sum_{k=1}^{m} \frac{p_{k}}{4 \Omega_{k}} \frac{1}{\left(\omega-\Omega_{k}\right)^{2}+\Gamma_{k}^{2}}
$$


with real fitting parameters $p_{k}, \Omega_{k}$ and $\Gamma_{k}$. This decomposition is not restricted to a any shape of the spectral density and can therefore be applied to approximate complicated band structures. This enables one to avoid the assumption of the wide-band limit and to take influences of the band structure on the dissipative electron transfer between the wire and the leads fully into account.

With the complex roots of the Fermi function and of function (8), the application of the theorem of residues results in

$$
\begin{gathered}
C_{12}(t)=\sum_{k=1}^{m} \frac{p_{k}}{4 \Omega_{k} \Gamma_{k}}\left(n_{F}\left(-\Omega_{k}^{-}+E_{F}\right) e^{-i \Omega_{k}^{-} t}\right)-\frac{2 i}{\beta} \sum_{k}^{m^{\prime}} J_{R}\left(\nu_{k}^{*}\right) e^{-i \nu_{k}^{*} t}=\sum_{k=1}^{m+m^{\prime}} a_{12}^{k} e^{\gamma_{12}^{k} t} \\
C_{21}(t)=\sum_{k=1}^{m} \frac{p_{k}}{4 \Omega_{k} \Gamma_{k}}\left(n_{F}\left(\Omega_{k}^{+}-E_{F}\right) e^{i \Omega_{k}^{+} t}\right)-\frac{2 i}{\beta} \sum_{k}^{m^{\prime}} J_{R}\left(\nu_{k}\right) e^{i \nu_{k} t}=\sum_{k=1}^{m+m^{\prime}} a_{21}^{k} e^{\gamma_{21}^{k} t}
\end{gathered}
$$

with the abbreviations $\Omega_{k}^{+}=\Omega_{k}+i \Gamma_{k}$ and $\Omega_{k}^{-}=\Omega_{k}-i \Gamma_{k}$ and the Matsubara frequencies $\nu_{k}=i \frac{2 \pi k+\pi}{\beta}+E_{F}$. Rigorously, the sum over the Matsubara frequencies would be infinite but it can be truncated at a finite $m^{\prime}$ depending on the temperature of the system $T$ and the spectral width of $J_{R}(\omega)$. The pure exponential dependence of the correlation function on time allows one to derive a set of differential equations for the auxiliary density operators

$$
\begin{gathered}
\frac{\partial}{\partial t} \Lambda_{x x^{\prime}}^{k}(t)=a_{x x^{\prime}}^{k} K_{x^{\prime}} \rho_{S}(t)-i\left[H_{S}(t), \Lambda_{x x^{\prime}}^{k}(t)\right]+\gamma_{x x^{\prime}}^{k} \Lambda_{x x^{\prime}}^{k}(t), \\
\frac{\partial}{\partial t} \widehat{\Lambda}_{x x^{\prime}}^{k}(t)=\left(a_{x^{\prime} x}^{k}\right)^{*} \rho_{S}(t) K_{x^{\prime}}-i\left[H_{S}(t), \widehat{\Lambda}_{x x^{\prime}}^{k}(t)\right]+\left(\gamma_{x^{\prime} x}^{k}\right)^{*} \widehat{\Lambda}_{x x^{\prime}}^{k}(t)
\end{gathered}
$$

with $\Lambda_{x x^{\prime}}(t)=\sum_{k=1}^{m+m^{\prime}} \Lambda_{x x^{\prime}}^{k}(t)$ and $\widehat{\Lambda}_{x x^{\prime}}(t)=\sum_{k=1}^{m+m^{\prime}} \widehat{\Lambda}_{x x^{\prime}}^{k}(t)$.

As detailed in Ref. [7] it is now easy to give an expression for the current. Using the electron number operator of the left lead with the summation performed over the reservoir degrees of freedom $N_{l}=\sum_{q} c_{q}^{\dagger} c_{q}$ yields

$$
I_{l}(t)=e \frac{\mathrm{d}}{\mathrm{d} t} \operatorname{tr}\left\{N_{l} \rho_{S}(t)\right\}=-i e \operatorname{tr}\left\{\left[N_{l}, H(t)\right] \rho_{S}(t)\right\}=2 e \operatorname{Re}\left(\operatorname{tr}_{S}\left\{c_{1}^{\dagger} \Lambda_{12}(t)-c_{1}^{\dagger} \widehat{\Lambda}_{12}(t)\right\}\right) .
$$

Numerical results. - In the following we set the value $0.1 \mathrm{eV}$ for the tight-binding hopping parameter $\Delta$. This parameter also constitutes the energy scale for the results shown. The system temperature is set to $T=0.25 \Delta / k_{B} \approx 290 \mathrm{~K}$. Though with the method described above one can model complex energy dependences in the wire-lead coupling, here we use for simplicity only one Lorentzian in the spectral density (8). The maximum strength is chosen to be $0.1 \Delta$. This is achieved by using $p_{1}=0.644 \mathrm{eV}, \Gamma_{1}=5.44 \mathrm{eV}$, and $\Omega_{1}=0.544 \mathrm{eV}$. With the chosen energy settings, a time unit in the system corresponds to $0.66 \mathrm{fs}$. The resulting current unit can be extracted from Eq. (13) and is equal to a macroscopic value of $1[e]=2.43 * 10^{-4} \mathrm{~A}$. The carrier frequency is set to $1 \mathrm{eV}$.

In Fig. 2 the phenomenon of CDT is shown for a monochromatic laser field of amplitude $A=2.405 \mathrm{eV}$. This amplitude corresponds to a full CDT $[7,16]$. The wire consists of two sites with on-site energies $E_{1}=E_{2}=0.54 \mathrm{eV}$ which are initially empty. The Fermi energy of the left lead is $1.0 \mathrm{eV}$ and that of the right one is $0.0 \mathrm{eV}$. The top panel of Fig. 2 shows the 


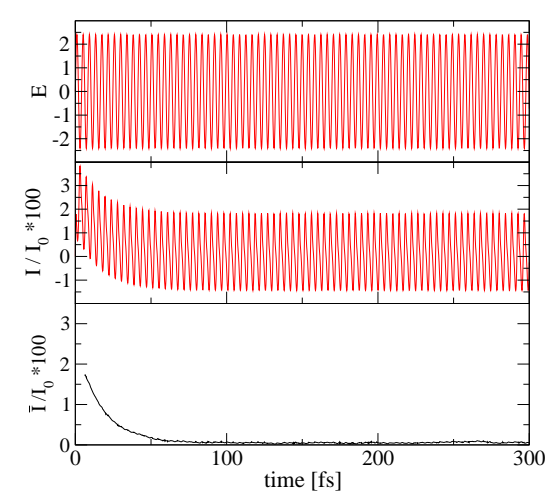

Fig. 2

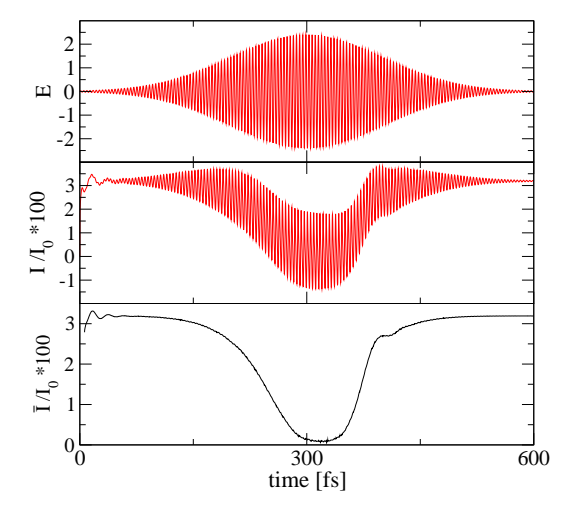

Fig. 3

Fig. 2 - The scenario of CDT for a monochromatic laser field. The top panel shows the laser field, the middle one the current through the wire, and the lowest panel the averaged current.

Fig. 3 - Same as in Fig. 2 but for a short laser pulse with length $\sigma=50$ fs.

laser field while the middle panel displays the time-dependent current $I(t)$ through the wire. To be able to see the effect more easily we also show an averaged time-dependent current $\bar{I}$ determined by averaging $I(t)$ over three periods of the highly-oscillating carrier field. Since the laser is instantaneously turned on at $t=0$, one sees some transient behavior during the first 150 fs before the CDT fully sets in. From Fig. 3 one can deduce that the transient behavior is mainly due to the slow onset of the CDT and not due to filling the wire sites. In this figure no laser pulse is applied to the wire for the first $150 \mathrm{fs}$ and one observes a transient behavior during the first $50 \mathrm{fs}$ due to filling the wire sites. Then the laser pulse with a Gaussian shape $E(t)=A \exp \left(-(t-T)^{2} /\left(2 \sigma^{2}\right)\right) \sin \left(\omega_{d} t\right)$ with $\sigma=50 \mathrm{fs}$ and a maximum amplitude as for the monochromatic laser before sets in. For the short laser pulse a complete suppression of the average current is not reached at any time but a dip in the current is clearly visible. The shape of this indentation does not show a Gaussian form nor is it symmetric. The current decays smoothly towards a minimum value while at the end of the laser pulse the current goes back to its equilibrium value showing some oscillations.

To investigate the dependence of the average current on the pulse length, the average

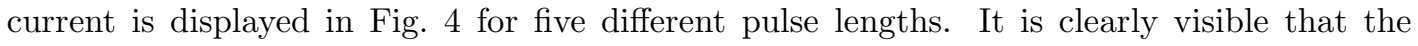
minimum average current approaches zero for longer pulse lengths. For an infinite pulse length the CDT condition would be perfectly fulfilled and the average current would vanish as shown in Fig. 2. It is interesting that already for $\sigma=5 \mathrm{fs}$ one can see a clear dip in the average current although only very few oscillations of the carrier field are involved in this scenario which reach an amplitude close to the maximum for that pulse length.

All the calculations above were performed for $U / \Delta=2$. To investigate the effect of electron correlation on the CDT we also did the calculations using a Gaussian laser pulse with $\sigma=50$ fs for different $U$. The results in Fig. 5 show already an interesting transient behavior of the current while the laser pulse is still off. Without correlation the equilibrium current has the largest value. Turning on correlation the equilibrium value of the average current decreases almost to the same value for electron attraction and repulsion of $U / \Delta= \pm 2$. For the larger 


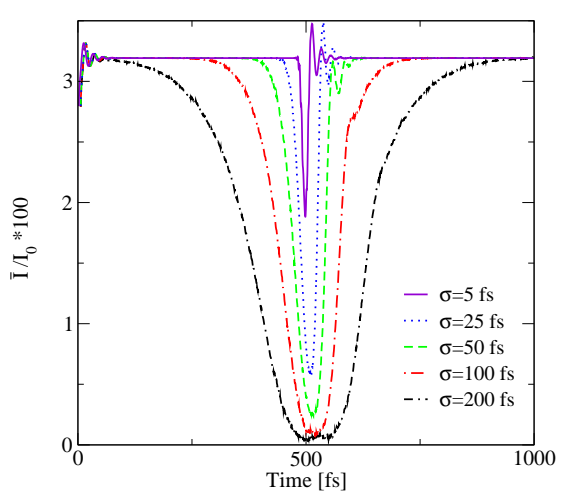

Fig. 4

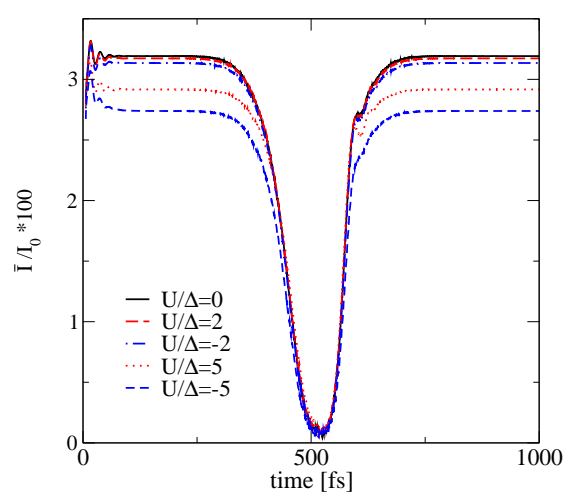

Fig. 5

Fig. 4 - The average current through a molecular wire for different pulse lengths. Other parameters are the same as in Fig. 3.

Fig. 5 - The effect of electron correlation for a pulse with length $\sigma=50 \mathrm{fs}$. Displayed are cases with no correlation, electron attraction and electron repulsion.

value of $U / \Delta= \pm 5$ the equilibrium value of the current is reduced much more for electron attraction than for repulsion. Interestingly enough, the minimum value of the current is nearly the same in all cases regardless of the strength and sign of correlation. So the degree of CDT depends on the length of the laser pulse but not on electron correlation.

Finally we want to show that effects of laser pulses are also visible if the conditions for CDT are not fulfilled. Then in general a much weaker suppression of the current is visible.

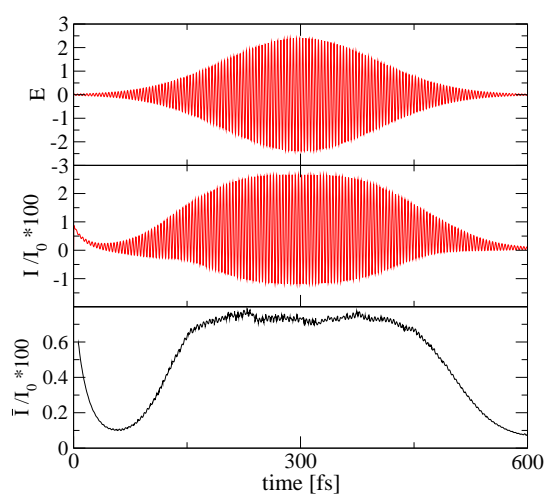

Fig. 6 - After a short transient behavior no net current would flow through the wire for $E_{F, l}=0.82$ $\mathrm{eV}, E_{F, r}=-0.82 \mathrm{eV}, E_{1}=-0.82 \mathrm{eV}$ and $E_{2}=1.36 \mathrm{eV}$. The laser pulse which is the same as in Fig. 3 shifts the levels of the wire in such a manner that a temporary current is possible. 
But if one uses a configuration in which, due to energetic reasons, a current is not possible but an external bias is applied, then there are cases in which the laser can initiate a current through the wire. This phenomenon can be seen in Fig. 6. The parameters are set so that the high energy level of the second wire site blocks the current. This creation of a current by a laser pulse does not seem to follow a pattern as the CDT. Its physical basis is quite different: The laser pulse brings the levels of the wire to a position which energetically allows for population transfer between the sites and between leads and outermost sites. So this effect can simply be explained by energetic reasoning and no interference effects are needed for an interpretation.

Summary and outlook. - The main point of this work is to show that the phenomenon of CDT exists in molecular wires also for short laser pulses and not only for monochromatic laser fields. Nevertheless the amplitude condition as for cw-laser fields plays an important role. If these conditions are not fulfilled only a very weak current suppression is achieved by the laser pulse. If the CDT condition is fulfilled the current can be suppressed almost completely already for short laser pulses. For longer pulses the current suppression gets more effective. Electron correlation was treated in a version for spinless fermions. Large correlation can have a rather large effect on the equilibrium current, larger for electron attraction than repulsion. Finally we showed that for parameter regions in which no equilibrium current occurs, a laser pulse can lead to a weak current caused by temporary shifting the energy levels of the wire into a position in which population transfer is possible.

In conclusion we showed interesting possibilities how ultra-short laser pulses can influence the current through molecular wires. Though the present calculations were performed for a simple model system, the basic physics should survive in more complex systems. Here we have restricted ourselves to a wire of only two sites although similar results can be obtained for longer wires. Using the effect of CDT with short laser pulses allows for the construction of fast opto-electronic switches. This opens, of course, a huge variety of applications for similar scenarios and possible technical devices even though a number of experimental hurdles have to be passed before one can think of direct technical applications.

\section{REFERENCES}

[1] Nitzan A. and Ratner M. A., Science, 300 (2003) 1384.

[2] Ghosh A. W., Damle P. S., Datta S., and Nitzan A., MRS Bulletin, 6 (2004) 391.

[3] Meir Y. and Wingreen N. S., Phys. Rev. Lett., 68 (1992) 2512.

[4] Kohler S., Lehmann J., and Hänggi P., Phys. Rep., 406 (2005) 379.

[5] Li X.-Q., Luo J.-Y., Yang Y.-G., Cui P., and Yan Y. J., Phys. Rev. B, 71 (2005) 205304.

[6] Ovchinnikov I. V. and Neuhauser D., J. Chem. Phys., 122 (2005) 024707.

[7] Welack S., Schreiber M., and Kleinekathöfer U., J. Chem. Phys., 124 (2006) 044712.

[8] Gerstner V., Knoll A., Pfeiffer W., Thon A., and Gerber G., J. Appl. Phys., 88 (2000) 4851.

[9] Meier C. and Tannor D. J., J. Chem. Phys., 111 (1999) 3365.

[10] Yan Y. J. and Xu R.-X., Ann. Rev. Phys. Chem., 56 (2005) 187.

[11] Kleinekathöfer U., J. Chem. Phys., 121 (2004) 2505.

[12] Welack S., Kleinekathöfer U., and Schreiber M., Laser-driven molecular wires studied by a non-markovian density matrix approach (2006). J. Lumin. (accepted).

[13] Grossmann F., Dittrich T., Jung P., and Hänggi P., Phys. Rev. Lett., 67 (1991) 516.

[14] Grossmann F. and HängGi P., Europhys. Lett., 18 (1992) 571.

[15] Stockburger J. T., Phys. Rev. E, 59 (1999) R4709.

[16] Lehmann J., Camalet S., Kohler S., and Hänggi P., Chem. Phys. Lett., 368 (2003) 282. 
EUROPHYSICS LETTERS

[17] Redfield A. G., IBM J. Res. Dev., 1 (1957) 19. 